PROCEEDINGS OF THE

AMERICAN MATHEMATICAL SOCIETY

Volume 133, Number 9, Pages 2671-2680

S 0002-9939(05)07781-6

Article electronically published on March 17, 2005

\title{
CALDERÓN-ZYGMUND OPERATORS ON HARDY SPACES WITHOUT THE DOUBLING CONDITION
}

\author{
WENGU CHEN, YAN MENG, AND DACHUN YANG
}

(Communicated by Andreas Seeger)

\begin{abstract}
Let $\mu$ be a non-negative Radon measure on $\mathbb{R}^{d}$ which only satisfies some growth condition. In this paper, the authors obtain the boundedness of Calderón-Zygmund operators in the Hardy space $H^{1}(\mu)$.
\end{abstract}

\section{INTRODUCTION}

In recent years, many papers focus on the analysis on $\mathbb{R}^{d}$ with non-doubling measure; see 2, 5, 6, 8, 3, 4, 1] and their references. Moreover, the analysis on such $\mathbb{R}^{d}$ was proved to play a striking role in solving the long open Painlevé's problem by Tolsa in [9]; see also [10] for more background of this. Throughout this paper, the Euclidean space $\mathbb{R}^{d}$ is endowed with a non-negative Radon measure $\mu$ which only satisfies the following growth condition that there exists $C_{0}>0$ such that

$$
\mu(B(x, r)) \leq C_{0} r^{n}
$$

for all $x \in \mathbb{R}^{d}$ and $r>0$, where $B(x, r)=\left\{y \in \mathbb{R}^{d}:|y-x|<r\right\}, n$ is a fixed number and $0<n \leq d$. Such a measure $\mu$ is not necessary to be doubling. We recall that $\mu$ is said to satisfy the doubling condition if there exists $C>0$ such that $\mu(B(x, 2 r)) \leq C \mu(B(x, r))$ for all $x \in \operatorname{supp}(\mu)$ and $r>0$. It is well known that the doubling condition in the analysis on spaces of homogeneous type is a key assumption. However, some research has now indicated that the doubling condition is superfluous for most of the classical Calderón-Zygmund theory.

Let $K$ be a function on $\mathbb{R}^{d} \times \mathbb{R}^{d} \backslash\{(x, y): x=y\}$ satisfying that for $x \neq y$,

$$
|K(x, y)| \leq C|x-y|^{-n} \text {, }
$$

and for $|x-y| \geq 2\left|x-x^{\prime}\right|$,

$$
\left|K(x, y)-K\left(x^{\prime}, y\right)\right|+\left|K(y, x)-K\left(y, x^{\prime}\right)\right| \leq C \frac{\left|x-x^{\prime}\right|^{\delta}}{|x-y|^{n+\delta}}
$$

where $\delta \in(0,1]$ and $C>0$ is a constant. The Calderón-Zygmund operator associated to the above kernel $K$ and the measure $\mu$ is formally defined by

$$
T f(x)=\int_{\mathbb{R}^{d}} K(x, y) f(y) d \mu(y) .
$$

Received by the editors March 8, 2004 and, in revised form, April 22, 2004.

2000 Mathematics Subject Classification. Primary 42B20; Secondary 42B30, 42B25, 43A99.

This project was supported by NNSF (No. 10271015 \& No. 10371080) of China and the third (corresponding) author was also supported by RFDP (No. 20020027004) of China. 
This integral may not be convergent for many functions. Thus we consider the truncated operators $T_{\varepsilon}$ for $\varepsilon>0$ defined by

$$
T_{\varepsilon} f(x)=\int_{|x-y|>\varepsilon} K(x, y) f(y) d \mu(y) .
$$

We say that $T$ is bounded on $L^{2}(\mu)$ if the operators $\left\{T_{\varepsilon}\right\}_{\varepsilon>0}$ are bounded on $L^{2}(\mu)$ uniformly on $\varepsilon>0$. In this case, there is an operator $\widetilde{T}$ which is the weak limit as $\varepsilon \rightarrow 0$ of some subsequence of operators $\left\{T_{\varepsilon}\right\}_{\varepsilon>0}$; see [5]. It is easy to see that $\widetilde{T}$ is still bounded on $L^{2}(\mu)$; moreover, for $f \in L^{2}(\mu)$ with compact support and a. e. $x \in \mathbb{R}^{d} \backslash \operatorname{supp}(f)$,

$$
\widetilde{T} f(x)=\int_{\mathbb{R}^{d}} K(x, y) f(y) d \mu(y)
$$

with the same $K$ as in (1.2) and (1.3). By the same argument of Tolsa as in [5, 7, we see that $\widetilde{T}$ is also bounded from $L^{1}(\mu)$ into weak- $L^{1}(\mu)$ and from $H^{1}(\mu)$ into $L^{1}(\mu)$.

In this paper, we will prove that $\widetilde{T}$ is bounded on the Hardy space $H^{1}(\mu)$ if $\widetilde{T}^{*} 1=0$. Here, by $\widetilde{T}^{*} 1=0$, we mean that for any bounded function $b$ with compact support and $\int_{\mathbb{R}^{d}} b d \mu=0$,

$$
\int_{\mathbb{R}^{d}} \tilde{T} b(x) d \mu(x)=0 .
$$

We remark that for such a function $b, b \in H^{1}(\mu)$ and therefore, $\widetilde{T} b \in L^{1}(\mu)$. Also, if $\widetilde{T} b \in H^{1}(\mu)$, then $\widetilde{T} b$ should satisfy (1.5) by the definition of the Hardy space $H^{1}(\mu)$; see [5, 8] or Definition 2 below. Thus, in some sense, the condition (1.5) is also necessary.

If $\mu$ is the $d$-dimensional Lebesgue measure on $\mathbb{R}^{d}$, this result is well known and it was proved by verifying that $\widetilde{T}$ maps any atom of the Hardy space $H^{1}\left(\mathbb{R}^{d}\right)$ into some molecule. However, if $\mu$ only satisfies (1.1), it is still unknown if there is a molecular characterization for the Hardy space $H^{1}(\mu)$. We will prove that $\widetilde{T}$ is bounded on the Hardy space $H^{1}(\mu)$ via its "grand" maximal function characterization of Tolsa in 8 and its new atomic characterization of the authors in [1].

Definition 1. Given $f \in L_{\text {loc }}^{1}(\mu)$, we set

$$
M_{\Phi} f(x)=\sup _{\varphi \sim x}\left|\int_{\mathbb{R}^{d}} f \varphi d \mu\right|,
$$

where the notation $\varphi \sim x$ means that $\varphi \in L^{1}(\mu) \cap C^{1}\left(\mathbb{R}^{d}\right)$ and satisfies

(i) $\|\varphi\|_{L^{1}(\mu)} \leq 1$,

(ii) $0 \leq \varphi(y) \leq \frac{1}{|y-x|^{n}}$ for all $y \in \mathbb{R}^{d}$, and

(iii) $|\nabla \varphi(y)| \leq \frac{1}{|y-x|^{n+1}}$ for all $y \in \mathbb{R}^{d}$, where $\nabla=\left(\partial / \partial x_{1}, \cdots, \partial / \partial x_{d}\right)$.

Based on Theorem 1.2 of Tolsa in [8], we define the Hardy space $H^{1}(\mu)$ as follows.

Definition 2. The Hardy space $H^{1}(\mu)$ is the set of all functions $f \in L^{1}(\mu)$ satisfying that $\int_{\mathbb{R}^{d}} f d \mu=0$ and $M_{\Phi} f \in L^{1}(\mu)$. Moreover, we define the norm of $f \in H^{1}(\mu)$ by

$$
\|f\|_{H^{1}(\mu)}=\|f\|_{L^{1}(\mu)}+\left\|M_{\Phi} f\right\|_{L^{1}(\mu)}
$$


Theorem 1. Let $K$ be the function on $\mathbb{R}^{d} \times \mathbb{R}^{d} \backslash\{(x, y): x=y\}$ satisfying (1.2) and (1.3). Suppose that the operator $\widetilde{T}$ in (1.4) is bounded on $L^{2}(\mu)$ and $\widetilde{T}^{*} 1=0$ as in (1.5). Then $\widetilde{T}$ is bounded on $H^{1}(\mu)$.

It is known that the dual space of $H^{1}(\mu)$ is the space $R B M O(\mu)$, which was introduced by Tolsa in [5]. From Theorem 1, the fact that $R B M O(\mu)=\left(H^{1}(\mu)\right)^{*}$ (see [5]) and a standard dual argument, it is easy to deduce the boundedness of the transpose operator of $\widetilde{T}$ in $R B M O(\mu)$ as below.

Corollary 1. Let $\widetilde{T}$ be the same as in Theorem 1 . Then $\widetilde{T}^{*}$, the transpose operator of $\widetilde{T}$, is bounded on $R B M O(\mu)$.

Remark 1. Obviously, from different subsequences of operators $\left\{T_{\varepsilon}\right\}_{\varepsilon>0}$ which are bounded on $L^{2}(\mu)$ uniformly on $\varepsilon>0$, one may deduce different $\widetilde{T}$ 's. However, they are all bounded on $L^{2}(\mu)$ and satisfy (1.4). But, the relation between these different $\widetilde{T}$ 's is still open.

In what follows, $C$ denotes a positive constant that is independent of the main parameters involved but whose value may differ from line to line.

\section{Proof of Theorem 1}

We begin with some necessary notation and definitions. Throughout this paper, we only consider the closed cubes with sides parallel to the coordinate axes. For any cube $Q$ and any $\alpha>0, \alpha Q$ denotes the cube with the same center as $Q$ and $l(\alpha Q)=\alpha l(Q)$, where $l(Q)$ denotes the side length of the cube $Q$.

Given two cubes $Q \subset R$ in $\mathbb{R}^{d}$, set

$$
K_{Q, R}=1+\sum_{k=1}^{N_{Q, R}} \frac{\mu\left(2^{k} Q\right)}{l\left(2^{k} Q\right)^{n}},
$$

where $N_{Q, R}$ is the smallest positive integer $k$ such that $l\left(2^{k} Q\right) \geq l(R)$; see [5] for some properties of $K_{Q, R}$.

To prove Theorem 1, we need to recall the atomic characterization of the Hardy space $H^{1}(\mu)$ as follows.

Definition 3. Let $\rho>1,1<p \leq \infty$ and $\gamma \in \mathbb{N}$. A function $b \in L_{\mathrm{loc}}^{1}(\mu)$ is called a $(p, \gamma)$-atomic block if

(1) there exists some cube $R$ such that $\operatorname{supp}(b) \subset R$,

(2) $\int_{\mathbb{R}^{d}} b d \mu=0$

(3) for $j=1,2$, there are functions $a_{j}$ supported on cube $Q_{j} \subset R$ and numbers $\lambda_{j} \in \mathbb{R}$ such that $b=\lambda_{1} a_{1}+\lambda_{2} a_{2}$, and

$$
\left\|a_{j}\right\|_{L^{p}(\mu)} \leq\left[\mu\left(\rho Q_{j}\right)\right]^{1 / p-1}\left[K_{Q_{j}, R}\right]^{-\gamma}
$$

Then we define

$$
|b|_{H_{\mathrm{atb}, \gamma}^{1, p}(\mu)}=\left|\lambda_{1}\right|+\left|\lambda_{2}\right| .
$$


We say that $f \in H_{\mathrm{atb}, \gamma}^{1, p}(\mu)$ if there are $(p, \gamma)$-atomic blocks $\left\{b_{i}\right\}_{i \in \mathbb{N}}$ such that

$$
f=\sum_{i=1}^{\infty} b_{i}
$$

with $\sum_{i=1}^{\infty}\left|b_{i}\right|_{H_{\mathrm{atb}, \gamma}^{1, p}(\mu)}<\infty$. The $H_{\mathrm{atb}, \gamma}^{1, p}(\mu)$ norm of $f$ is defined by

$$
\|f\|_{H_{\mathrm{atb}, \gamma}^{1, p}(\mu)}=\inf \left\{\sum_{i}\left|b_{i}\right|_{H_{\mathrm{atb}, \gamma}^{1, p}(\mu)}\right\},
$$

where the infimum is taken over all the possible decompositions of $f$ into $(p, \gamma)$ atomic blocks.

The above definition when $\gamma=1$ was introduced by Tolsa in [5] and when $\gamma>1$ by the authors in [1]. It was proved in [5, 1] that the definition of $H_{\mathrm{atb}, \gamma}^{1, p}(\mu)$ is independent of the chosen constant $\rho>1$, and for any integer $\gamma \geq 1$ and $1<p \leq$ $\infty$, all the atomic Hardy spaces $H_{\text {atb, } \gamma}^{1, p}(\mu)$ are just the Hardy space $H^{1}(\mu)$ with equivalent norms. We remark that in the proof of Theorem 1 below, we need to choose $\gamma>1$, especially, $\gamma=2$.

The following lemma will be used in the proof of Theorem 1 .

Lemma 1. Let $M_{\Phi}$ be as in Definition 1 and $1<p<\infty$. Then $M_{\Phi}$ is bounded on $L^{p}(\mu)$.

In fact, Tolsa proved that $M_{\Phi}$ is bounded from $H^{1}(\mu)$ into $L^{1}(\mu)$; see Lemma 3.1 in [8]. On the other hand, it is obvious that $M_{\Phi}$ is bounded on $L^{\infty}(\mu)$. By Theorem 7.2 in [5], we obtain that $M_{\Phi}$ is bounded on $L^{p}(\mu)$ for $1<p<\infty$.

Now we turn to the proof of Theorem 1.

Proof of Theorem 1. By a standard argument, it suffices to verify that for any atomic block $b$ as in Definition 3 with $\rho=4, p=\infty$ and $\gamma=2, \widetilde{T} b$ is in $H^{1}(\mu)$ with norm $C|b|_{H_{a t b, 2}^{1, \infty}(\mu)}$, where $C$ is independent of $b$. Let all the notation be the same as in Definition 3. By our choices, $a_{j}$ now satisfies the following size condition that

$$
\left\|a_{j}\right\|_{L^{\infty}(\mu)} \leq\left[\mu\left(4 Q_{j}\right) K_{Q_{j}, R}^{2}\right]^{-1}
$$

where $j=1,2$.

The assumption that $\widetilde{T}^{*} 1=0$ tells us that $\int_{\mathbb{R}^{d}} \widetilde{T} b d \mu=0$. Recalling that $\widetilde{T}$ is bounded from $H^{1}(\mu)$ into $L^{1}(\mu)$ (see [5] ), we obtain

$$
\|\widetilde{T} b\|_{L^{1}(\mu)} \leq C|b|_{H_{a t b, 2}^{1, \infty}(\mu)} .
$$

By this and Definition 2, we deduce that the proof of Theorem 1 can be reduced to proving that

$$
\left\|M_{\Phi}(\widetilde{T} b)\right\|_{L^{1}(\mu)} \leq C|b|_{H_{a t b, 2}^{1, \infty}(\mu)} .
$$

Write

$$
\left\|M_{\Phi}(\widetilde{T} b)\right\|_{L^{1}(\mu)}=\int_{4 R} M_{\Phi}(\widetilde{T} b)(x) d \mu(x)+\int_{\mathbb{R}^{d} \backslash 4 R} M_{\Phi}(\widetilde{T} b)(x) d \mu(x)=\mathrm{I}+\mathrm{II} .
$$


Noting that $M_{\Phi}$ is sublinear, we can control I by

$$
\mathrm{I} \leq \int_{4 R} M_{\Phi}\left[(\widetilde{T} b) \chi_{8 R}\right](x) d \mu(x)+\int_{4 R} M_{\Phi}\left[(\widetilde{T} b) \chi_{\mathbb{R}^{d} \backslash 8 R}\right](x) d \mu(x)=\mathrm{I}_{1}+\mathrm{I}_{2} .
$$

From the fact that for $j=1,2, Q_{j} \subset R$, it follows that for any $z \in Q_{j}$ and any $y \in 2^{k+1} R \backslash 2^{k} R, k \geq 3,|y-z| \geq l\left(2^{k-2} R\right)$. By this fact, (ii) of Definition 1, (1.2) and (2.1), we obtain

$$
\begin{aligned}
\mathrm{I}_{2} & \leq \int_{4 R} \sup _{\varphi \sim x}\left[\int_{\mathbb{R}^{d} \backslash 8 R}|\widetilde{T} b(y)| \varphi(y) d \mu(y)\right] d \mu(x) \\
& \leq \sum_{j=1}^{2}\left|\lambda_{j}\right| \int_{4 R} \sum_{k=3}^{\infty} \int_{2^{k+1} R \backslash 2^{k} R}\left|\int_{Q_{j}} K(y, z) a_{j}(z) d \mu(z)\right| \frac{1}{|x-y|^{n}} d \mu(y) d \mu(x) \\
& \leq C \sum_{j=1}^{2}\left|\lambda_{j}\right| \sum_{k=3}^{\infty}\left\|a_{j}\right\|_{L^{\infty}(\mu)} \mu\left(Q_{j}\right) \frac{\mu\left(2^{k+1} R\right)}{l\left(2^{k-2} R\right)^{n}} \frac{\mu(4 R)}{l\left(2^{k-2} R\right)^{n}} \\
& \leq C \sum_{j=1}^{2}\left|\lambda_{j}\right| .
\end{aligned}
$$

To estimate $\mathrm{I}_{1}$, we write

$$
\begin{aligned}
\mathrm{I}_{1} \leq & \sum_{j=1}^{2}\left|\lambda_{j}\right| \int_{4 Q_{j}} M_{\Phi}\left[\left(\widetilde{T} a_{j}\right) \chi_{8 R}\right](x) d \mu(x) \\
& +\sum_{j=1}^{2}\left|\lambda_{j}\right| \int_{4 R \backslash 4 Q_{j}} M_{\Phi}\left[\left(\widetilde{T} a_{j}\right) \chi_{2 Q_{j}}\right](x) d \mu(x) \\
& +\sum_{j=1}^{2}\left|\lambda_{j}\right| \int_{4 R \backslash 4 Q_{j}} M_{\Phi}\left[\left(\widetilde{T} a_{j}\right) \chi_{8 R \backslash 2 Q_{j}}\right](x) d \mu(x) \\
= & \mathrm{I}_{11}+\mathrm{I}_{12}+\mathrm{I}_{13} .
\end{aligned}
$$

The Hölder inequality, Lemma 1 , the boundedness of $\widetilde{T}$ in $L^{2}(\mu)$ and (2.1) lead to

$$
\begin{aligned}
\mathrm{I}_{11} & \leq \sum_{j=1}^{2}\left|\lambda_{j}\right| \mu\left(4 Q_{j}\right)^{1 / 2}\left\|M_{\Phi}\left[\left(\widetilde{T} a_{j}\right) \chi_{8 R}\right]\right\|_{L^{2}(\mu)} \\
& \leq C \sum_{j=1}^{2}\left|\lambda_{j}\right| \mu\left(4 Q_{j}\right)^{1 / 2}\left\|\widetilde{T} a_{j}\right\|_{L^{2}(\mu)} \\
& \leq C \sum_{j=1}^{2}\left|\lambda_{j}\right| \mu\left(4 Q_{j}\right)^{1 / 2}\left\|a_{j}\right\|_{L^{2}(\mu)} \\
& \leq C \sum_{j=1}^{2}\left|\lambda_{j}\right| \mu\left(4 Q_{j}\right)\left\|a_{j}\right\|_{L^{\infty}(\mu)} \\
& \leq C \sum_{j=1}^{2}\left|\lambda_{j}\right| .
\end{aligned}
$$


For $j=1,2$, denote $N_{Q_{j}, 4 R}$ simply by $N_{j}$. By (ii) of Definition 1 , the Hölder inequality, the boundedness of $\widetilde{T}$ in $L^{2}(\mu)$ and (2.1), we have

$$
\begin{aligned}
\mathrm{I}_{12} & \leq \sum_{j=1}^{2}\left|\lambda_{j}\right| \sum_{k=2}^{N_{j}} \int_{2^{k+1} Q_{j} \backslash 2^{k} Q_{j}} \sup _{\varphi \sim x}\left|\int_{2 Q_{j}} \widetilde{T} a_{j}(y) \varphi(y) d \mu(y)\right| d \mu(x) \\
& \leq \sum_{j=1}^{2}\left|\lambda_{j}\right| \sum_{k=2}^{N_{j}} \int_{2^{k+1} Q_{j} \backslash 2^{k} Q_{j}} \frac{1}{l\left(2^{k-2} Q_{j}\right)^{n}} d \mu(x) \int_{2 Q_{j}}\left|\widetilde{T} a_{j}(y)\right| d \mu(y) \\
& \leq \sum_{j=1}^{2}\left|\lambda_{j}\right| \sum_{k=2}^{N_{j}} \frac{\mu\left(2^{k+1} Q_{j}\right)}{l\left(2^{k-2} Q_{j}\right)^{n}}\left\|\widetilde{T} a_{j}\right\|_{L^{2}(\mu)} \mu\left(2 Q_{j}\right)^{1 / 2} \\
& \leq C \sum_{j=1}^{2}\left|\lambda_{j}\right| K_{Q_{j}, R} \mu\left(2 Q_{j}\right)^{1 / 2}\left\|a_{j}\right\|_{L^{2}(\mu)} \\
& \leq C \sum_{j=1}^{2}\left|\lambda_{j}\right|
\end{aligned}
$$

where we have used the fact that

$$
K_{Q_{j}, 4 R} \leq C K_{Q_{j}, R}
$$

For $\mathrm{I}_{13}$, we further decompose it into

$$
\begin{aligned}
\mathrm{I}_{13}= & \sum_{j=1}^{2}\left|\lambda_{j}\right| \sum_{k=2}^{N_{j}} \int_{2^{k+1} Q_{j} \backslash 2^{k} Q_{j}} M_{\Phi}\left[\left(\widetilde{T} a_{j}\right) \chi_{8 R \backslash 2 Q_{j}}\right](x) d \mu(x) \\
\leq & \sum_{j=1}^{2}\left|\lambda_{j}\right| \sum_{k=2}^{N_{j}} \int_{2^{k+1} Q_{j} \backslash 2^{k} Q_{j}} M_{\Phi}\left[\left|\widetilde{T} a_{j}\right| \chi_{2^{k+2} Q_{j} \backslash 2^{k-1} Q_{j}}\right](x) d \mu(x) \\
& +\sum_{j=1}^{2}\left|\lambda_{j}\right| \sum_{k=2}^{N_{j}} \int_{2^{k+1} Q_{j} \backslash 2^{k} Q_{j}} M_{\Phi}\left[\left|\widetilde{T} a_{j}\right| \chi_{\left.\max \left\{2^{k+2} Q_{j}, 8 R\right\} \backslash 2^{k+2} Q_{j}\right](x) d \mu(x)}\right. \\
& +\sum_{j=1}^{2}\left|\lambda_{j}\right| \sum_{k=2}^{N_{j}} \int_{2^{k+1} Q_{j} \backslash 2^{k} Q_{j}} M_{\Phi}\left[\left|\widetilde{T} a_{j}\right| \chi_{2^{k-1} Q_{j} \backslash 2 Q_{j}}\right](x) d \mu(x) \\
= & \mathrm{E}+\mathrm{F}+\mathrm{G} .
\end{aligned}
$$

Lemma 1, (1.2) and (2.1) tell us that

$$
\begin{aligned}
& \leq \sum_{j=1}^{2}\left|\lambda_{j}\right| \sum_{k=2}^{N_{j}} \mu\left(2^{k+1} Q_{j}\right)^{1 / 2}\left\|M_{\Phi}\left[\left|\widetilde{T} a_{j}\right| \chi_{2^{k+2} Q_{j} \backslash 2^{k-1} Q_{j}}\right]\right\|_{L^{2}(\mu)} \\
\leq & C \sum_{j=1}^{2}\left|\lambda_{j}\right| \sum_{k=2}^{N_{j}} \mu\left(2^{k+1} Q_{j}\right)^{1 / 2} \\
& \quad \times\left\{\int_{2^{k+2} Q_{j} \backslash 2^{k-1} Q_{j}}\left|\int_{Q_{j}} K(y, z) a_{j}(z) d \mu(z)\right|^{2} d \mu(y)\right\}^{1 / 2} \\
\leq & C \sum_{j=1}^{2}\left|\lambda_{j}\right| \sum_{k=2}^{N_{j}} \frac{\mu\left(2^{k+2} Q_{j}\right)}{l\left(2^{k-3} Q_{j}\right)^{n}}\left\|a_{j}\right\|_{L^{\infty}(\mu)} \mu\left(Q_{j}\right) \\
\leq & C \sum_{j=1}^{2}\left|\lambda_{j}\right| .
\end{aligned}
$$


By (ii) of Definition 1, (1.2), (2.3) and (2.1), we easily see that

$$
\begin{aligned}
\mathrm{G} & \leq \sum_{j=1}^{2}\left|\lambda_{j}\right| \sum_{k=2}^{N_{j}} \int_{2^{k+1} Q_{j} \backslash 2^{k} Q_{j}} \sup _{\varphi \sim x}\left[\int_{2^{k-1} Q_{j} \backslash 2 Q_{j}}\left|\widetilde{T} a_{j}(y)\right| \varphi(y) d \mu(y)\right] d \mu(x) \\
& \leq \sum_{j=1}^{2}\left|\lambda_{j}\right| \sum_{k=2}^{N_{j}} \int_{2^{k+1} Q_{j} \backslash 2^{k} Q_{j}} \sum_{l=1}^{k-2} \int_{2^{l+1} Q_{j} \backslash 2^{l} Q_{j}}\left|\int_{Q_{j}} K(y, z) a_{j}(z) d \mu(z)\right| \\
& \leq C \sum_{j=1}^{2}\left|\lambda_{j}\right| \sum_{k=2}^{N_{j}} \frac{\mu\left(2^{k+1} Q\right)}{|y-x|^{n}} d \mu(y) d \mu(x) \\
& \leq C \sum_{j=1}^{2}\left|\lambda_{j}\right|\left[K_{Q_{j}, R}\right]^{2}\left\|a_{j}\right\|_{L^{\infty}(\mu)} \mu\left(Q_{j}\right) \\
& \leq C \sum_{j=1}^{2}\left|\lambda_{j}\right| .
\end{aligned}
$$

An argument similar to the estimate for G leads to

$$
\mathrm{F} \leq C \sum_{j=1}^{2}\left|\lambda_{j}\right| .
$$

The estimates for $\mathrm{E}, \mathrm{F}$ and $\mathrm{G}$ give the desired estimate for $\mathrm{I}_{13}$. Combining the estimates for $\mathrm{I}_{11}, \mathrm{I}_{12}, \mathrm{I}_{13}$ and $\mathrm{I}_{2}$ yields

$$
\mathrm{I}=\int_{4 R} M_{\Phi}(\widetilde{T} b)(x) d \mu(x) \leq C \sum_{j=1}^{2}\left|\lambda_{j}\right|=C|b|_{H_{a t b, 2}^{1, \infty}(\mu)}
$$

Now we turn to the estimate for II. Let $x_{R}$ be the center of the cube $R$. Invoking that $\widetilde{T}^{*} 1=0$, we obtain

$$
\begin{aligned}
\mathrm{II}= & \int_{\mathbb{R}^{d} \backslash 4 R} \sup _{\varphi \sim x}\left|\int_{\mathbb{R}^{d}} \widetilde{T} b(y)\left[\varphi(y)-\varphi\left(x_{R}\right)\right] d \mu(y)\right| d \mu(x) \\
\leq & \int_{\mathbb{R}^{d} \backslash 4 R \varphi \sim x} \sup _{\varphi}\left|\int_{2 R} \widetilde{T} b(y)\left[\varphi(y)-\varphi\left(x_{R}\right)\right] d \mu(y)\right| d \mu(x) \\
& \quad+\int_{\mathbb{R}^{d} \backslash 4 R} \sup _{\varphi \sim x}\left|\int_{\mathbb{R}^{d} \backslash 2 R} \widetilde{T} b(y)\left[\varphi(y)-\varphi\left(x_{R}\right)\right] d \mu(y)\right| d \mu(x) \\
= & \mathrm{II}_{1}+\mathrm{II}_{2} .
\end{aligned}
$$

Note that for any $z \in 2 R, x \in 2^{k+1} R \backslash 2^{k} R$, and $k \geq 2$, we have $|x-z| \geq l\left(2^{k-2} R\right)$. This together with (iii) of Definition 1 and the mean value theorem leads to

$$
\left|\varphi(y)-\varphi\left(x_{R}\right)\right| \leq C \frac{l(R)}{l\left(2^{k-2} R\right)^{n+1}}
$$


for $y \in 2 R$. By $(2.5),(1.2)$, the Hölder inequality, the boundedness of $\widetilde{T}$ in $L^{2}(\mu)$ and (2.1), we have

$$
\begin{aligned}
\mathrm{II}_{1} \leq & \sum_{j=1}^{2}\left|\lambda_{j}\right| \sum_{k=2}^{\infty} \int_{2^{k+1} R \backslash 2^{k} R} \sup _{\varphi \sim x}\left[\int_{2 R \backslash 2 Q_{j}}\left|\widetilde{T} a_{j}(y)\right|\left|\varphi(y)-\varphi\left(x_{R}\right)\right| d \mu(y)\right] d \mu(x) \\
& +\sum_{j=1}^{2}\left|\lambda_{j}\right| \sum_{k=2}^{\infty} \int_{2^{k+1} R \backslash 2^{k} R} \sup _{\varphi \sim x}\left[\int_{2 Q_{j}}\left|\widetilde{T} a_{j}(y)\right|\left|\varphi(y)-\varphi\left(x_{R}\right)\right| d \mu(y)\right] d \mu(x) \\
\leq & C \sum_{j=1}^{2}\left|\lambda_{j}\right| \sum_{k=2}^{\infty} \int_{2^{k+1} R \backslash 2^{k} R} \frac{l(R)}{l\left(2^{k-2} R\right)^{n+1}} \\
& \times \sum_{l=1}^{N_{j}-1} \int_{2^{l+1} Q_{j} \backslash 2^{l} Q_{j}} \int_{Q_{j}} \frac{\left|a_{j}(z)\right|}{|y-z|^{n}} d \mu(z) d \mu(y) d \mu(x) \\
& +C \sum_{j=1}^{2}\left|\lambda_{j}\right| \sum_{k=2}^{\infty} \int_{2^{k+1} R \backslash 2^{k} R} \frac{l(R)}{l\left(2^{k-2} R\right)^{n+1}}\left\|\left(\widetilde{T} a_{j}\right) \chi_{2 Q_{j}}\right\|_{L^{1}(\mu)} d \mu(x) \\
\leq & C \sum_{j=1}^{2}\left|\lambda_{j}\right| \sum_{k=2}^{\infty} 2^{-k} \sum_{l=1}^{N_{j}-1} \frac{\mu\left(2^{l+1} Q_{j}\right)}{l\left(2^{l+1} Q_{j}\right)^{n}}\left\|a_{j}\right\|_{L^{\infty}(\mu)} \mu\left(Q_{j}\right) \\
& +C \sum_{j=1}^{2}\left|\lambda_{j}\right| \sum_{k=2}^{\infty} 2^{-k}\left\|\left(\widetilde{T} a_{j}\right) \chi_{2 Q_{j}}\right\|_{L^{2}(\mu)} \mu\left(2 Q_{j}\right)^{1 / 2} \\
\leq & C \sum_{j=1}^{2}\left|\lambda_{j}\right| K_{Q_{j}, R}\left\|a_{j}\right\|_{L^{\infty}(\mu)} \mu\left(Q_{j}\right)+C \sum_{j=1}^{2}\left|\lambda_{j}\right|\left\|a_{j}\right\|_{L^{2}(\mu)} \mu\left(2 Q_{j}\right)^{1 / 2} \\
\leq & C \sum_{j=1}^{2}\left|\lambda_{j}\right| .
\end{aligned}
$$

We further estimate $\mathrm{II}_{2}$ by

$$
\begin{aligned}
& \mathrm{II}_{2}=\sum_{k=2}^{\infty} \int_{2^{k+1} R \backslash 2^{k} R} \sup _{\varphi \sim x}\left|\int_{\mathbb{R}^{d} \backslash 2 R} \widetilde{T} b(y)\left[\varphi(y)-\varphi\left(x_{R}\right)\right] d \mu(y)\right| d \mu(x) \\
& \leq \sum_{k=2}^{\infty} \int_{2^{k+1} R \backslash 2^{k} R} M_{\Phi}\left[|\tilde{T} b| \chi_{2^{k+2} R \backslash 2^{k-1} R}\right](x) d \mu(x) \\
& +\sum_{k=2}^{\infty} \int_{2^{k+1} R \backslash 2^{k} R} \sup _{\varphi \sim x}\left[\int_{2^{k+2} R \backslash 2^{k-1} R}|\widetilde{T} b(y)| \varphi\left(x_{R}\right) d \mu(y)\right] d \mu(x) \\
& \left.+\sum_{k=2}^{\infty} \int_{2^{k+1} R \backslash 2^{k} R \varphi \sim x} \sup _{\varphi \mathbb{R}^{d} \backslash 2^{k+2} R}|\widetilde{T} b(y)|\left\{\varphi(y)+\varphi\left(x_{R}\right)\right\} d \mu(y)\right] d \mu(x) \\
& +\sum_{k=2}^{\infty} \int_{2^{k+1} R \backslash 2^{k} R} \sup _{\varphi \sim x}\left[\int_{2^{k-1} R \backslash 2 R}|\widetilde{T} b(y)|\left\{\varphi(y)+\varphi\left(x_{R}\right)\right\} d \mu(y)\right] d \mu(x) \\
& =\mathrm{II}_{21}+\mathrm{II}_{22}+\mathrm{II}_{23}+\mathrm{II}_{24} \text {. }
\end{aligned}
$$


From Lemma 1, the fact that $\int_{\mathbb{R}^{d}} b d \mu=0$ and (1.3), we can deduce that

$$
\begin{aligned}
\mathrm{II}_{21} \leq & \sum_{k=2}^{\infty} \mu\left(2^{k+1} R\right)^{1 / 2}\left\|M_{\Phi}\left[|\widetilde{T} b| \chi_{2^{k+2} R \backslash 2^{k-1} R}\right]\right\|_{L^{2}(\mu)} \\
\leq & C \sum_{k=2}^{\infty} \mu\left(2^{k+1} R\right)^{1 / 2} \\
& \times\left\{\int_{2^{k+2} R \backslash 2^{k-1} R}\left|\int_{R}\left[K(y, z)-K\left(y, x_{R}\right)\right] b(z) d \mu(z)\right|^{2} d \mu(y)\right\}^{1 / 2} \\
\leq & C \sum_{k=2}^{\infty} \mu\left(2^{k+1} R\right) \frac{l(R)^{\delta}}{l\left(2^{k} R\right)^{n+\delta}}\|b\|_{L^{1}(\mu)} \\
\leq & C \sum_{j=1}^{2}\left|\lambda_{j}\right|,
\end{aligned}
$$

where we have used the fact that

$$
\|b\|_{L^{1}(\mu)} \leq \sum_{j=1}^{2}\left|\lambda_{j}\right|\left\|a_{j}\right\|_{L^{1}(\mu)} \leq C \sum_{j=1}^{2}\left|\lambda_{j}\right| .
$$

An argument similar to the estimate for $\mathrm{II}_{21}$ tells us that

$$
\mathrm{II}_{22} \leq C \sum_{j=1}^{2}\left|\lambda_{j}\right| .
$$

Finally, we estimate $\mathrm{II}_{23}$. By the fact that $\int_{\mathbb{R}^{d}} b d \mu=0$, (ii) of Definition 1 and (1.3), we obtain

$$
\begin{aligned}
\mathrm{II}_{23} \leq & \sum_{k=2}^{\infty} \int_{2^{k+1} R \backslash 2^{k} R} \sum_{l=k+2}^{\infty} \int_{2^{l+1} R \backslash 2^{l} R} \int_{R}\left|K(y, z)-K\left(y, x_{R}\right) \| b(z)\right| d \mu(z) \\
& \times\left[\frac{1}{|y-x|^{n}}+\frac{1}{\left|x_{R}-x\right|^{n}}\right] d \mu(y) d \mu(x) \\
\leq & C \sum_{k=2}^{\infty} \sum_{l=k+2}^{\infty} \frac{\mu\left(2^{k+1} R\right)}{l\left(2^{k+1} R\right)^{n}} \frac{\mu\left(2^{l+1} R\right) l(R)^{\delta}}{l\left(2^{l+1} R\right)^{n+\delta}}\|b\|_{L^{1}(\mu)} \\
\leq & C \sum_{j=1}^{2}\left|\lambda_{j}\right| .
\end{aligned}
$$

An argument similar to the estimate for $\mathrm{II}_{23}$ indicates that

$$
\mathrm{II}_{24} \leq C \sum_{j=1}^{2}\left|\lambda_{j}\right| .
$$

Combining the estimates for $\mathrm{II}_{21}, \mathrm{II}_{22}, \mathrm{II}_{23}$ and $\mathrm{II}_{24}$, we obtain the desired estimate for $\mathrm{II}_{2}$. The estimates for $\mathrm{II}_{1}$ and $\mathrm{II}_{2}$ tell us that

$$
\mathrm{II}=\int_{\mathbb{R}^{d} \backslash 4 R} M_{\Phi}(\widetilde{T} b)(x) d \mu(x) \leq C|b|_{H_{a t b, 2}^{1, \infty}(\mu)} .
$$

The estimates (2.4) and (2.6) lead to (2.2), and this completes the proof of our theorem. 


\section{ACKNOWLedGement}

The authors would like to express their deep thanks to the referee and Professor Andreas Seeger for their several valuable remarks and suggestions which made this article more readable.

\section{REFERENCES}

[1] G. Hu, Y. Meng and D. Yang, New atomic characterization of $H^{1}$ space with non-doubling measures and its applications, Math. Proc. Camb. Phil. Soc. to appear.

[2] F. Nazarov, S. Treil and A. Volberg, Cauchy integral and Calderón-Zygmund operators on nonhomogeneous spaces, Internat. Math. Res. Notices 15 (1997), 703-726. MR1470373 (99e:42028)

[3] F. Nazarov, S. Treil and A. Volberg, Accretive system Tb-theorems on nonhomogeneous spaces, Duke Math. J. 113 (2002), 259-312. MR.1909219 (2003g:42030)

[4] F. Nazarov, S. Treil and Volberg, The Tb-theorem on non-homogeneous spaces, Acta Math. 190 (2003), 151-239. MR1998349

[5] X. Tolsa, BMO, $H^{1}$ and Calderón-Zygmund operators for non doubling measures, Math. Ann. 319 (2001), 89-149. MR.1812821 (2002c:42029)

[6] X. Tolsa, Littlewood-Paley theory and the T(1) theorem with non-doubling measures, Adv. Math. 164 (2001), 57-116. MR1870513 (2003e:42029)

[7] X. Tolsa, A proof of the weak $(1,1)$ inequality for singular integrals with non doubling measures based on a Calderón-Zygmund decomposition, Publ. Mat. 45 (2001), 163-174. MR:1829582(2002d:42019)

[8] X. Tolsa, The space $H^{1}$ for nondoubling measures in terms of a grand maximal operator, Trans. Amer. Math. Soc. 355 (2003), 315-348. MR1928090 (2003e:42030)

[9] X. Tolsa, Painlevé's problem and the semiadditivity of analytic capacity, Acta Math. 190 (2003), 105-149. MR1982794

[10] J. Verdera, The fall of the doubling condition in Calderón-Zygmund theory, Publ. Mat. 2002, Vol. Extra, 275-292. MR1964824 (2004b:42035)

Institute of Applied Physics and Computational Mathematics, P.O. 8009, Beijing, 100088, People's Republic of China

E-mail address: chenwg@mail.iapcm.ac.cn

School of Mathematical Sciences, Beijing Normal University, Beijing, 100875, PeoPLE'S RePublic of ChinA

E-mail address: mengyan@mail.bnu.edu.cn

School of Mathematical Sciences, Beijing Normal University, Beijing, 100875, PeoPLE'S Republic of ChinA

E-mail address: dcyang@bnu.edu.cn 